\title{
ON ALMOST AUTOMORPHIC SOLUTIONS OF LINEAR OPERATIONAL-DIFFERENTIAL EQUATIONS
}

\author{
GASTON M. N'GUÉRÉKATA
}

Received 20 January 2003

\begin{abstract}
We prove almost periodicity and almost automorphy of bounded solutions of linear differential equations $x^{\prime}(t)=A x(t)+f(t)$ for some class of linear operators acting in a Banach space.
\end{abstract}

2000 Mathematics Subject Classification: 34G10, 34K14.

1. Introduction. Throughout this note, $X$ will be a Banach space equipped with the norm topology and $X^{*}$ its dual space. $\langle\cdot, \cdot\rangle$ will denote the duality between $X$ and $X^{*}$.

We recall that a (strongly) continuous function $f: \mathbb{R} \rightarrow X$ is said to be almost automorphic if for every sequence of real numbers $\left(s_{n}^{\prime}\right)$, there exists a subsequence $\left(s_{n}\right)$ such that

$$
\begin{aligned}
& \lim _{n \rightarrow \infty} f\left(t+s_{n}\right)=g(t), \\
& \lim _{n \rightarrow \infty} g\left(t-s_{n}\right)=f(t),
\end{aligned}
$$

pointwise on $\mathbb{R}$.

$f$ is said to be weakly almost automorphic if (1.1) and (1.2) are replaced, respectively, by

$$
\begin{aligned}
& \text { weak- } \lim _{n \rightarrow \infty} f\left(t+s_{n}\right)=g(t) \text {, } \\
& \text { weak-limg } g\left(t-s_{n}\right)=f(t) \text {, }
\end{aligned}
$$

for each $t \in \mathbb{R}$.

When convergence in (1.1) and (1.2) is uniform in $t \in \mathbb{R}, f$ is said to be (Bochner) almost periodic. Almost periodic functions are characterized by the following so-called Bochner's criterion.

$f: \mathbb{R} \rightarrow X$ is almost periodic if and only if for every sequence of real numbers $\left(s_{n}^{\prime}\right)$, there exists a subsequence $\left(s_{n}\right)$ such that $\left(f\left(t+s_{n}\right)\right)$ is uniformly convergent in $t \in \mathbb{R}$.

For more information on almost automorphic and almost periodic functions, see, for instance, [2, 4].

We consider both differential equations

$$
\begin{aligned}
& x^{\prime}(t)=A x(t), \quad t \in \mathbb{R}, \\
& x^{\prime}(t)=A x(t)+f(t), \quad t \in \mathbb{R} .
\end{aligned}
$$


Recently, we proved in [1] that if $A$ is the infinitesimal generator of a $C_{0}$-group of bounded linear operators, then every solution of (1.3) with a relatively compact range in $X$ is almost periodic. In Section 2 below, we will investigate the case in which $A$ is a nilpotent operator, that is, there exists a natural number $n$ such that $A^{n}=\theta$. In Section 3, we deal with (1.4) where $A$ is of simplest type (see definition below). We prove that if $f$ is almost automorphic, then every solution is almost automorphic too. We also give a property of (1.5) when $A$ generates a $C_{0}$-group of bounded linear operators.

2. We first recall a result by Zaidman [4].

Proposition 2.1. If $A$ is nilpotent, then every solution of (1.1) is constant.

Now we state and prove the following result.

THEOREM 2.2. If $A$ is nilpotent, then every solution of (1.1) with a relatively compact range in $X$ is almost periodic.

Proof. Let $x(t)$ be a solution of (1.1) with a relatively compact range in $X$. Then, it is bounded. So, by the result above, it is constant over $\mathbb{R}$, that is,

$$
\|x(t)\|=\|x(0)\|, \quad t \in \mathbb{R} .
$$

Fix $s \in \mathbb{R}$ and consider $y_{s}(t)=x(t+s), t \in \mathbb{R}$. Then, $y_{s}(t)$ is also a bounded solution of (1.1), so $\left\|y_{s}(t)\right\|=\left\|y_{s}(0)\right\|$ for all $t \in \mathbb{R}$. Now fix $s_{1}, s_{2}$ in $\mathbb{R}$. Then, $y_{s_{1}}(t)-y_{s_{2}}(t)$ is a bounded solution of (1.1), so,

$$
\left\|y_{s_{1}}(t)-y_{s_{2}}(t)\right\|=\left\|y_{s_{1}}(0)-y_{s_{2}}(0)\right\|
$$

which gives

$$
\left\|x\left(t+s_{1}\right)-x\left(t+s_{2}\right)\right\|=\left\|x\left(s_{1}\right)-x\left(s_{2}\right)\right\| .
$$

Consider a given sequence $\left(s_{n}^{\prime}\right)$ in $\mathbb{R}$. Since $x(t)$ has relatively compact range in $X$, then there exists $\left(s_{n}\right) \subset\left(s_{n}^{\prime}\right)$ such that $\left(x\left(s_{n}\right)\right)$ is convergent, thus Cauchy. Given $\epsilon>0$, there exists $N$ such that $\left\|x\left(s_{n}\right)-x\left(s_{m}\right)\right\|<\epsilon$, if $n, m>N$ so that for every $t \in \mathbb{R}$,

$$
\left\|x\left(t+s_{n}\right)-x\left(t+s_{m}\right)\right\|<\epsilon
$$

which proves that $x(t)$ is almost periodic.

THEOREM 2.3. In a reflexive Banach Space $X$, if $A$ is nilpotent, then every bounded solution of (1.1) is weakly almost periodic.

The proof is based on the fact that in a reflexive Banach space, every sequence which is bounded in norm has weakly convergent subsequent (see [3, Theorem 1, page 26]). We apply the second part of the proof of the previous theorem to $\langle\phi, x(t)\rangle$ (arbitrary $\left.\phi \in X^{*}\right)$ to complete the proof. 
3. It is known that if $A \in £(X)$, where $X$ is a finite-dimensional space and $f$ is almost automorphic, then every bounded solution of (1.6) is almost automorphic [2]. We generalize this result here for a uniformly convex Banach space $X$ and $A$ of simplest type, that is, $A \in £(X)$ and

$$
A=\sum_{j=1}^{n} \alpha_{j} P_{j},
$$

where $\alpha_{j} \in \mathbb{C}, j=1, \ldots, n$, are mutually distinct and $P_{j}$ form a complete system (i.e., $\sum_{j=1}^{n} P_{j}=I$ ) of pairwise disjoint operators in $X$ with $P_{i} P_{j}=\delta_{i j} P_{i}$. We state and prove the following theorem.

THEOREM 3.1. If $A$ is of simplest type, then every bounded solution of (1.6) is almost automorphic.

Proof. If $x(t)$ is a solution, then $x(t)=\sum_{j=1}^{n} x_{j}(t)$, where $x_{j}(t)=P_{j}(x(t)), j=$ $1,2, \ldots, n$. We show that each $x_{j}(t)$ is almost automorphic. In fact,

$$
\begin{aligned}
x_{j}(t) & =P_{j} A x(t)+P_{j} f(t) \\
& =P_{j}\left(\sum_{i=1}^{n} \alpha_{i} P_{i}\right) x(t)+P_{j} f(t) \\
& =\alpha_{j} x_{j}(t)+P_{j} f(t),
\end{aligned}
$$

where $P_{j} f(t)$ is almost automorphic. Therefore, $x_{j}(t)$ is almost automorphic (see [2, page 69]) and so is $x(t)$. The proof is complete.

It is known that if $A$ is bounded linear operator on a Banach space $X$ and the function $x(t)=e^{t A} x_{0}$ is almost automorphic for some $x_{0} \in D(A)$, then either $\inf _{t \in \mathbb{R}}\|x(t)\|>0$ or $x(t)=0$, for every $t \in \mathbb{R}$. This is presented in [2, Theorem 2.1.9, page 17]. We now state a more general result.

THEOREM 3.2. Suppose $(T(t))_{t \in \mathbb{R}}$ is a $C_{0}$-group of bounded linear operators in a Banach space $X$ and the function $x(t)=T(t) x_{0}: \mathbb{R} \rightarrow X$ is weakly almost automorphic for some $x_{0} \in X$. Then, either $\inf _{t \in \mathbb{R}}\|x(t)\|>0$, or $x(t)=0$ for every $t \in \mathbb{R}$.

Proof. We assume that $\inf _{t \in \mathbb{R}}\|x(t)\|=0$. Then, we find a minimizing sequence of real numbers $\left(s_{n}^{\prime}\right)$ such that $s_{n}^{\prime} \rightarrow \infty$ and $\left\|s_{n}^{\prime}\right\| \rightarrow 0$ as $n \rightarrow \infty$.

Since $x(t)$ is weakly almost automorphic, there exists a subsequence $\left(s_{n}\right)$ of $\left(s_{n}^{\prime}\right)$ such that (1.3) and (1.4) are pointwise in $t \in \mathbb{R}$. And so, for every $\phi \in X^{*}$, we have

$$
\begin{aligned}
& \lim _{n \rightarrow \infty}\left\langle\phi, x\left(t+s_{n}\right)\right\rangle=\langle\phi, y(t)\rangle, \\
& \lim _{n \rightarrow \infty}\left\langle\phi, y\left(t-s_{n}\right)\right\rangle=\langle\phi, x(t)\rangle
\end{aligned}
$$

for each $t \in \mathbb{R}$. It is

$$
x\left(t+s_{n}\right)=T\left(t+s_{n}\right) x_{0}=T(t) T\left(s_{n}\right) x_{0}=T(t) x\left(s_{n}\right),
$$


$t \in \mathbb{R}, n=1,2, \ldots$, so that

$$
\lim _{n \rightarrow \infty}\left\langle\phi, T(t) x\left(s_{n}\right)\right\rangle=\langle\phi, y(t)\rangle
$$

for each $t \in \mathbb{R}, \phi \in X^{*}$. But

$$
\left|\left\langle\phi, T(t) x\left(s_{n}\right)\right\rangle\right| \leq\|\phi\|\|T(t)\|\left\|x\left(s_{n}\right)\right\| \rightarrow 0
$$

as $n \rightarrow \infty$. That implies $\langle\phi, y(t)\rangle=0$, for each $\phi \in X^{*}$ and $t \in \mathbb{R}$. So, $y(t)=0, t \in \mathbb{R}$, and consequently $x(t)=0, t \in \mathbb{R}$. The proof is now complete.

Application 3.3. Consider (1.5), where $A$ is the infinitesimal generator of a $C_{0^{-}}$ group of bounded linear operators $T(t), t \in \mathbb{R}$. Then, every mild weakly almost automorphic solution $x(t)$ satisfies the property: $\inf _{t \in \mathbb{R}}\|x(t)\|>0$, or $x(t)=0$ for every $t \in \mathbb{R}$.

Proof. Mild solutions of (1.6) in this case are written as $x(t)=T(t) x(0), t \in \mathbb{R}$. Then apply Theorem 3.2.

\section{REFERENCES}

[1] T. Diagana and G. M. N'Guérékata, On some perturbations of some abstract differential equations, Comment. Math. Prace Mat. 43 (2003), no. 2, 201-206.

[2] G. M. N'Guérékata, Almost Automorphic and Almost Periodic Functions in Abstract Spaces, Kluwer Academic/Plenum Publishers, New York, 2001.

[3] K. Yosida, Functional Analysis, Springer-Verlag, New York, 1974.

[4] S. Zaidman, Topics in Abstract Differential Equations. II, Pitman Research Notes in Mathematics Series, vol. 321, John Wiley \& Sons, New York, 1995.

Gaston M. N'Guérékata: Department of Mathematics, Morgan State University, Baltimore, MD 21251, USA

E-mail address: gnguerek@morgan.edu 


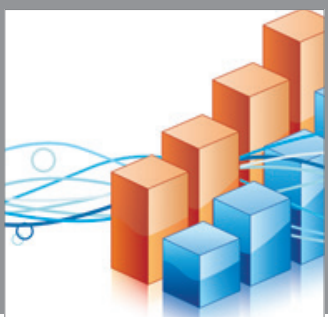

Advances in

Operations Research

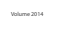

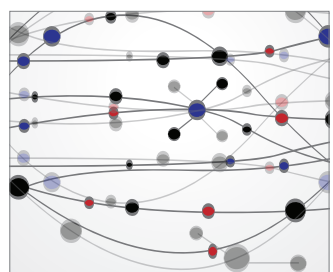

\section{The Scientific} World Journal
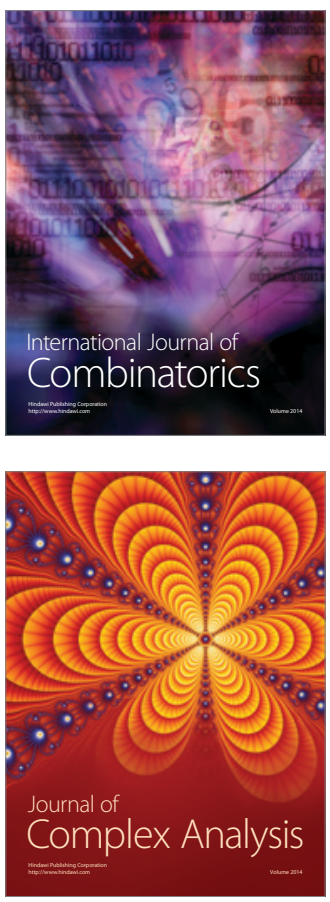

International Journal of

Mathematics and

Mathematical

Sciences
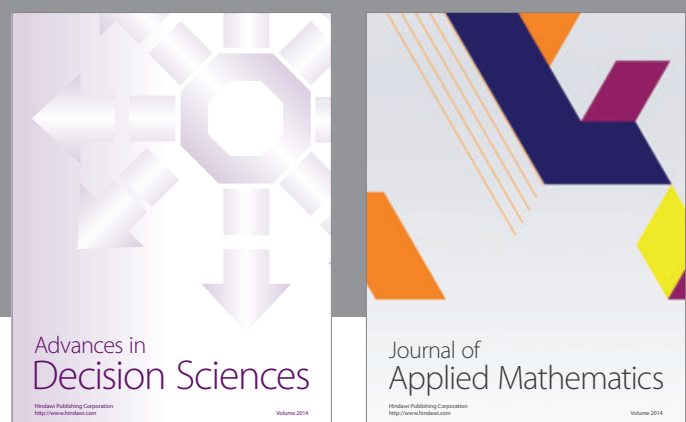

Journal of

Applied Mathematics
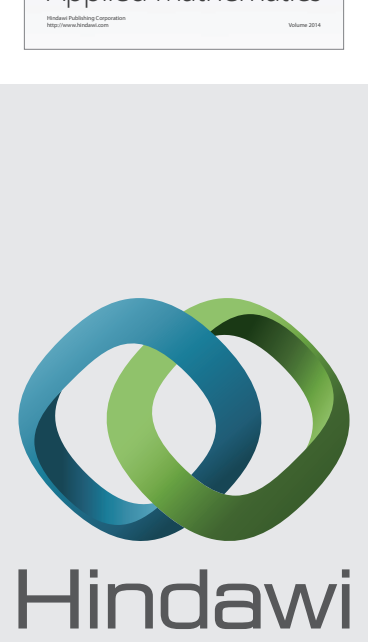

Submit your manuscripts at http://www.hindawi.com
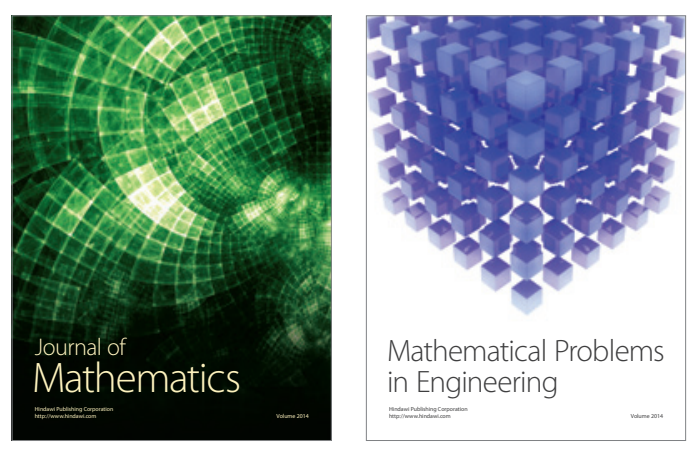

Mathematical Problems in Engineering
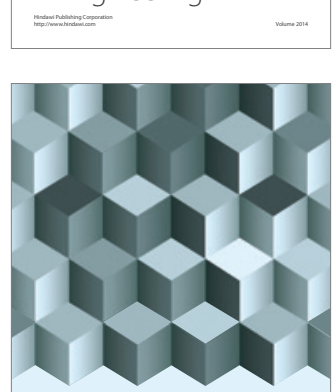

Journal of

Function Spaces
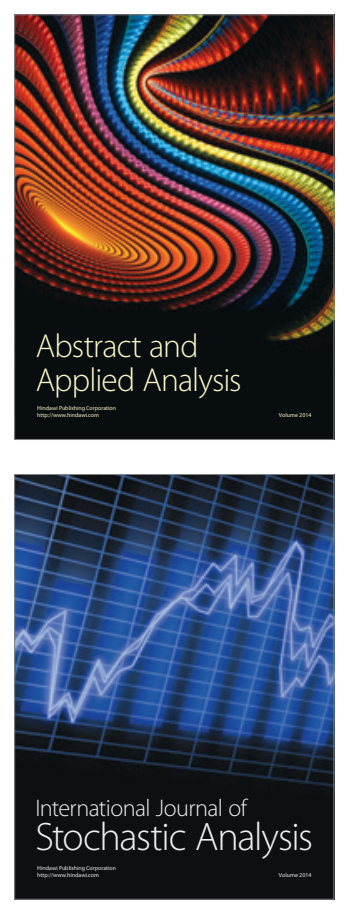

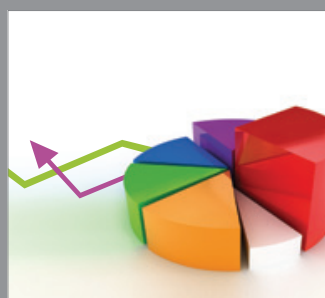

ournal of

Probability and Statistics

Promensencen
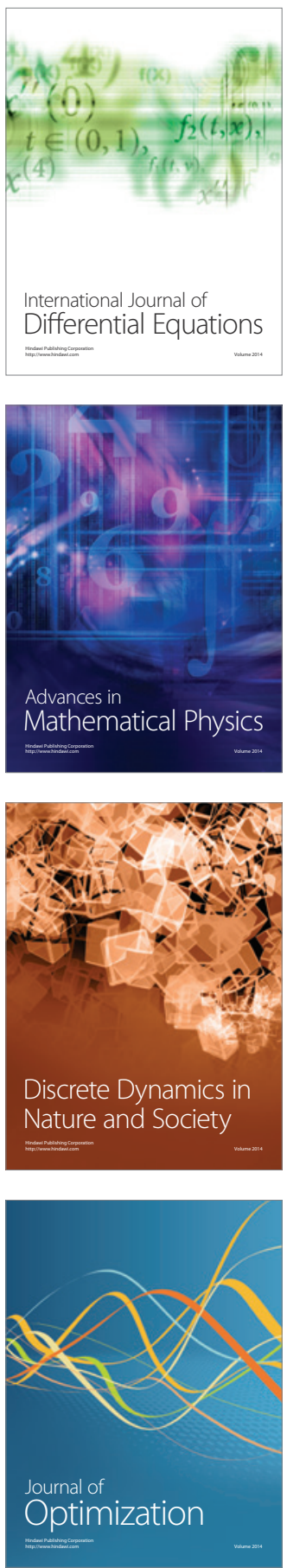